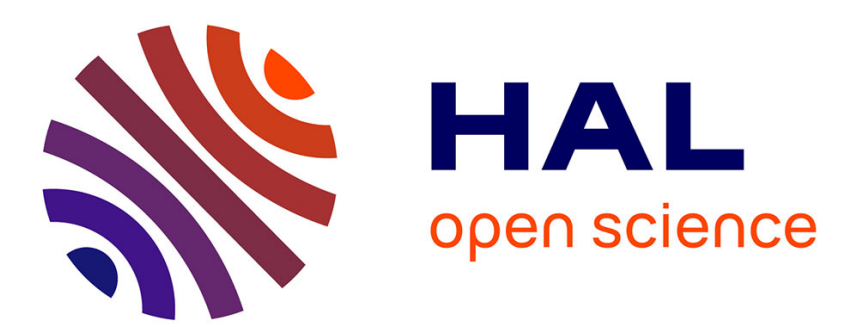

\title{
Détermination des caractéristiques de seuil de jonctions Josephson utilisées dans les dispositifs logiques à commande magnétique
}

\author{
G. Angenieux, J. Chilo, C. Monllor
}

\section{- To cite this version:}

G. Angenieux, J. Chilo, C. Monllor. Détermination des caractéristiques de seuil de jonctions Josephson utilisées dans les dispositifs logiques à commande magnétique. Revue de Physique Appliquée, 1985, 20 (2), pp.93-98. 10.1051/rphysap:0198500200209300 . jpa-00245311

HAL Id: jpa-00245311

https://hal.science/jpa-00245311

Submitted on 1 Jan 1985

HAL is a multi-disciplinary open access archive for the deposit and dissemination of scientific research documents, whether they are published or not. The documents may come from teaching and research institutions in France or abroad, or from public or private research centers.
L'archive ouverte pluridisciplinaire HAL, est destinée au dépôt et à la diffusion de documents scientifiques de niveau recherche, publiés ou non, émanant des établissements d'enseignement et de recherche français ou étrangers, des laboratoires publics ou privés. 


\title{
Détermination des caractéristiques de seuil de jonctions Josephson utilisées dans les dispositifs logiques à commande magnétique
}

\author{
G. Angenieux, J. Chilo et C. Monllor \\ Laboratoire d'Electromagnétisme, ENSERG, 23, rue des Martyrs, 38031 Grenoble, France
}

(Reçu le 30 janvier 1984, révisé les 12 juin et 11 octobre, accepté le 25 octobre 1984)

\begin{abstract}
Résumé. - Dans les dispositifs logiques Josephson à couplage magnétique, les effets dimensionnels et de proximité se manifestent par une inhomogénéité du champ magnétique de commande. Nous présentons une méthode numérique permettant de calculer les caractéristiques de seuil des jonctions, compte-tenu de ces effets. Nous mettons en évidence un relèvement des lobes secondaires et un lissage des caractéristiques. Ces phénomènes réduisent l'immunité au bruit des dispositifs logiques. La comparaison entre les résultats théoriques et expérimentaux nous permet également de calculer la valeur moyenne réelle du champ magnétique dans la jonction.
\end{abstract}

\begin{abstract}
In the magnetically coupled Josephson logic devices, dimensional and proximity effects provoke an inhomogeneity of the command magnetic field. We present a numerical method which allows us to compute the threshold characteristics of the junctions by taking these effects into account. We point out an increase of the side lobes and a smoothing of the characteristics. These phenomena reduce the noise immunity of logic devices. The comparison between computation results and experiments allows us to calculate the real mean value of the magnetic field in the junction.
\end{abstract}

\section{Introduction.}

Les dispositifs à jonctions Josephson associent un temps de commutation réduit $(10 \mathrm{ps})$ à une faible dissipation énergétique $(0,1 \mathrm{fJ})$. De plus, les interconnexions sont très peu dissipatives et dispersives, même pour des signaux très rapides [1] puisqu'elles sont supraconductrices. Les hautes performances que l'on peut attendre de cette technologie laissent prévoir son développement dans le domaine des ordinateurs ultra-rapides.

Dans les dispositifs Josephson, les niveaux logiques correspondent à l'état électrique des jonctions (état supraconducteur ou résistif pour une même valeur du courant de polarisation). Le changement d'état s'effectue lorsque le courant qui traverse la jonction devient supérieur à une valeur maximale $I_{g_{\max }}$ définissant le courant de seuil.

Deux techniques sont utilisées pour déclencher la commutation de l'état supraconducteur à l'état résistif [2] :

- l'injection directe consistant à superposer une impulsion de commande au courant de polarisation,

- le couplage magnétique qui permet d'abaisser le courant de seuil de la jonction par application d'un champ magnétique créé par une ou plusieurs lignes situées au-dessus de la jonction.

Le gain des dispositifs à couplage direct est plus important que celui des dispositifs à couplage magnétique. Cependant ces derniers présentent une excellente isolation électrique entre la sortie et l'entrée.

Dans les circuits modernes, les deux techniques sont souvent utilisées simultanément en disposant un étage d'isolement à couplage magnétique et un étage de gain à couplage direct [3].

Les travaux présentés ci-après concernent les dispositifs à couplage purement magnétique.

La figure 1 représente la section transversale d'un circuit typique constitué de trois lignes de commande, d'une jonction Josephson et d'un plan de masse.

Les relations entre le courant de seuil $I_{\mathrm{g}_{\max }}$ de la jonction et les courants de commande $I_{c}$ dans les trois lignes constituent les caractéristiques de seuil du dispositif.

La conception de fonctions logiques nécessite la connaissance précise de ces caractéristiques. Pour les calculer, il faut résoudre le système d'équations (1) à (3) [2] ;

$$
\begin{gathered}
\nabla^{2} \phi(y, z)=\frac{\sin \phi(y, z)}{\lambda_{\mathbf{J}}^{2}} \begin{array}{c}
\text { (équation de Sine Gordon } \\
\text { indépendante du temps) }
\end{array} \\
\nabla \phi(y, z)=\frac{2 \pi d \mu_{0}}{\Phi_{0}}\left(\mathbf{H} \times \mathbf{n}_{x}\right) \\
j(y, z)=j_{0} \sin \phi(y, z)
\end{gathered}
$$




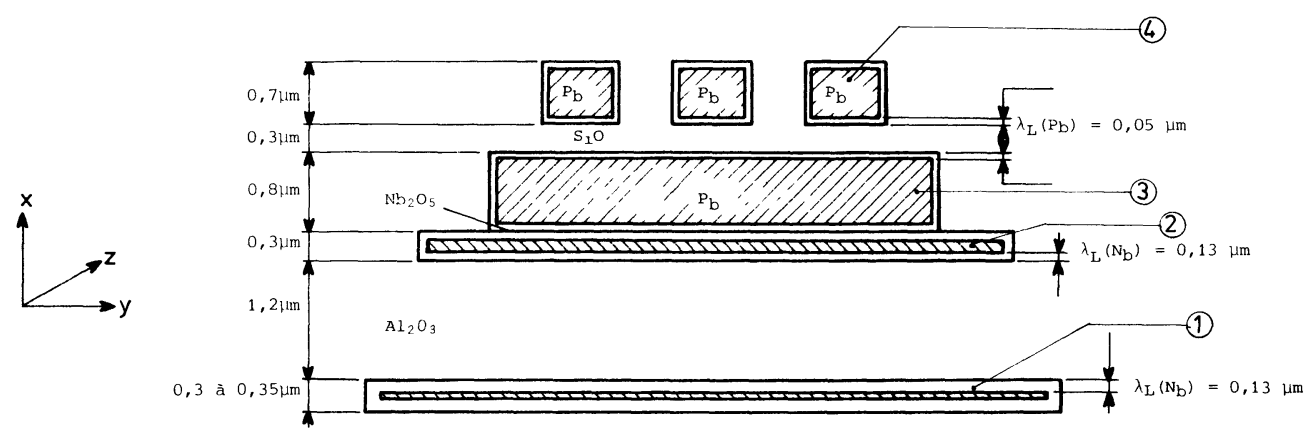

Fig. 1. - Géométrie typique d'une porte à trois entrées. (1) Plan de masse. (2) Electrode de base. (3) Contre-électrode. (4) Lignes de commande.

[Typical geometry of a three input gate. (1) Ground plane. (2) Base electrode. (3) Counter-electrode. (4) Command lines.]

où $\lambda_{\mathbf{J}}=$ profondeur de pénétration de Josephson; $d=a+\lambda_{1}+\lambda_{2} ; a$ : épaisseur de barrière de la jonction; $\lambda_{1}$ et $\lambda_{2}$ : profondeurs de pénétration de London $\lambda_{\mathrm{L}}$ relatives aux électrodes de la jonction; $\Phi_{0}:$ quantum de flux $\left(2,07 \times 10^{-15} \mathrm{~Wb}\right) ; j_{0}$ : densité de courant critique Josephson; $\nabla^{2}$ : Laplacien; $\nabla$ : Gradient.

Dans le cas général, la résolution analytique de l'équation non linéaire (1) est très complexe. L'approche habituelle consiste à considérer le champ magnétique uniforme dans la jonction, c'est-à-dire à négliger les effets de bord et de pénétration dans la jonction ainsi que les effets de proximité.

Cette hypothèse s'écrit :

$$
\begin{gathered}
\mathbf{H}_{\mathrm{c}}=\mathbf{H}_{\mathrm{c} \boldsymbol{y}} \\
\frac{\partial\left|\mathbf{H}_{\mathrm{c}}\right|}{\partial \boldsymbol{y}}=0
\end{gathered}
$$

où $\mathbf{H}_{\mathbf{c}}$ est le champ créé par les lignes (champ de commande). Dans ces conditions les relations (1) à (3) deviennent scalaires :

$$
\begin{gathered}
\frac{\partial^{2} \phi(z)}{\partial z^{2}}=\frac{\sin \phi(z)}{\lambda_{\mathrm{J}}^{2}} \\
\frac{\partial \phi(z)}{\partial z}=\frac{2 \pi d \mu_{0}}{\Phi_{0}} H_{y}(z) \\
j(z)=j_{0} \sin \phi(z) .
\end{gathered}
$$

La géométrie du dispositif fixe les conditions aux limites. En fonction de celles-ci plusieurs méthodes de résolution sont utilisables : lorsque la longueur $L$ des jonctions est inférieure à $2 \lambda$ (Fig. 3), il est possible de calculer analytiquement le courant de seuil [5]; pour les jonctions plus longues, il faut utiliser une résolution graphique [4] ou numérique [6]. Lorsque le champ magnétique appliqué est faible $\left(H_{\mathrm{c}}<2 \lambda_{\mathrm{J}} j_{0}\right)$ une linéarisation de l'équation (6) donne de bons résultats [7].

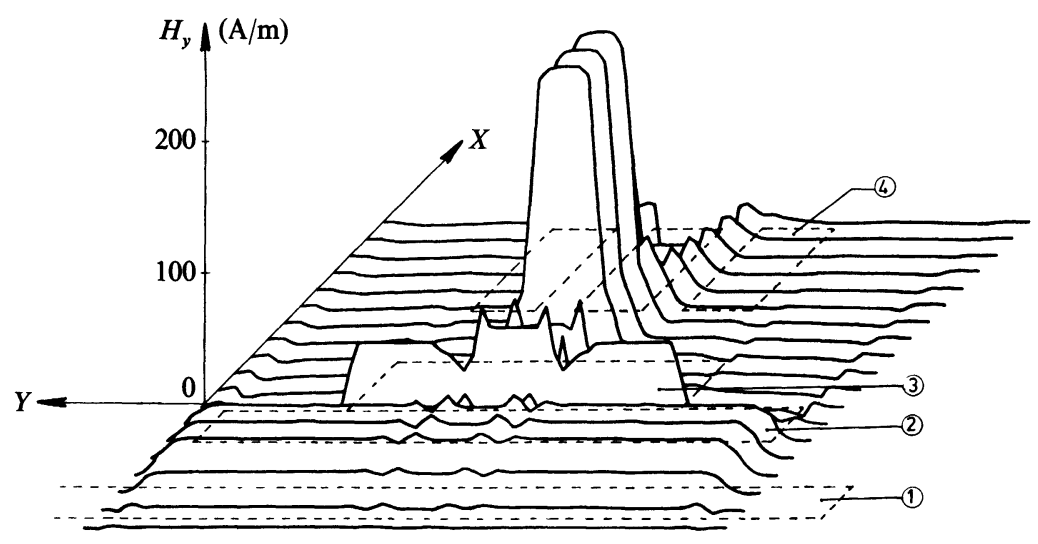

Fig. 2. - Champ magnétique tangentiel calculé par la méthode énergétique avec une excitation par la ligne centrale $(I=$ $1 \mathrm{~mA}$ ) pour le dispositif multicommandé de la figure 1. (1) Plan de masse. (2) Electrode de base. (3) Contre-électrode. (4) Ligne de commande.

[Tangential magnetic field calculated by the energetic method for the device of the figure 1. Current of $1 \mathrm{~mA}$ in the central line. (1) Ground plane. (2) Base electrode. (3) Counter-electrode. (4) Command line.] 


\section{Influence de la géométrie sur la structure du champ magnétique.}

Le champ magnétique est fonction des distributions des densités de courant dans la structure. Lorsque les épaisseurs des lignes de commande sont du même ordre de grandeur que $\lambda_{\mathrm{L}}$, les courants ne sont pas confinés en surface. Les effets dimensionnels (effets de bord) et la présence des autres conducteurs (effets de proximité) ont alors une grande influence sur la distribution de la densité de courant. Dans un article précédent [8], nous avons proposé une méthode énergétique permettant le calcul des distributions de courant et de champ magnétique en tout point d'une section transversale du dispositif. Par cette méthode nous mettons en évidence une importante variation transversale de la composante tangentielle $H_{y}$ du champ magnétique de commande (Fig. 2) et l'apparition d'une composante normale $H_{x}$ due aux effets de bord. Ceci montre que le calcul du courant de seuil de la jonction ne peut s'effectuer à partir du système d'équations (6), (7), (8) puisque les hypothèses (4) et (5) ne sont pas vérifiées. Il faut résoudre le système complet des équations (1), (2) et (3). Pour que celles-ci soient compatibles entre elles, il faut tenir compte de toutes les composantes de champ, y compris $H_{z}$.

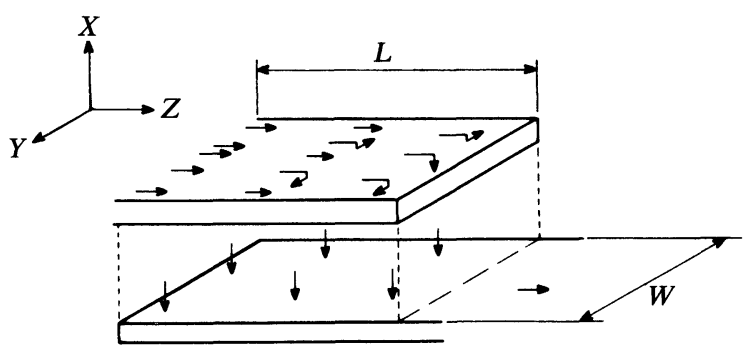

Fig. 3. - Allure des lignes de courant dans la contreélectrode.

[Aspect of the current lines in the counter-electrode.]

L'origine physique de cette composante est un courant transversal de "diffusion ", de densité $j_{y}$, prenant naissance dans la contre-électrode [9]. Cette situation est illustrée par la figure 3 représentant l'allure des lignes de courant dans la contre-électrode. Le phénomène en question est dû à la différence de répartition des courants dans les électrodes situées de part et d'autre de la barrière de la jonction [10] comme l'illustre la figure 4.

\section{Méthode de résolution.}

La jonction est divisée longitudinalement en éléments de largeur $\Delta y$ suffisamment faible pour pouvoir négliger les variations transversales du champ magné- tique sur chaque jonction élémentaire. Dans ces conditions, les distributions de courant Josephson dans les éléments peuvent se calculer indépendemment à partir des équations simplifiées (6), (7) et (8). Le courant total est obtenu en intégrant la densité de courant sur la surface de la jonction. Le courant de seuil $I_{g_{\max }}$ étant le courant maximum admissible par la jonction dans l'état supraconducteur, il faut rechercher dans l'ensemble des solutions des équations de Josephson celles qui maximisent le courant Josephson.

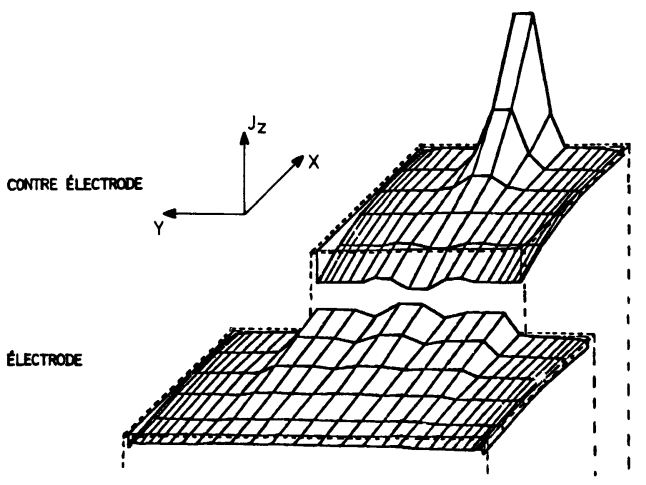

Fig. 4. - Densités du courant longitudinal $j_{z}$ induit de part et d'autre de la contre-électrode et dans l'électrode de base par un courant de $1 \mathrm{~mA}$ circulant dans la ligne centrale de commande.

[Longitudinal current densities $j_{z}$ induced in the counterelectrode and in the base electrode by a command current of $1 \mathrm{~mA}$ in the central line.]

Cette maximisation s'effectue par rapport à la phase à l'origine de la jonction $\phi_{(z=0)}$ car c'est le paramètre qui détermine la distribution de densité de courant Josephson [2]. La caractéristique de seuil est obtenue en effectuant ce calcul pour chaque valeur de courant de commande.

2.1 CAS GÉNÉRAL. - Le champ magnétique créé par le courant dans la jonction (champ propre) n'est pas négligeable devant le champ de commande. L'intégration de l'équation (7) est difficile et la valeur optimale de la phase à l'origine $\phi_{(z=0)}$ est très sensible à la longueur de la jonction et au champ de commande. Pour résoudre les équations, nous appliquons une méthode numérique itérative [6] à chaque jonction élémentaire. Cette méthode consiste à établir une relation de récurrence sur la valeur de la phase $\phi_{(z=0)}$ à partir de l'équation discrétisée. La récurrence est initialisée à partir de l'équation (7). Le courant de seuil est obtenu par l'équation (8) après maximisation par rapport à $\phi_{(z=0)}$. Ces étapes de calcul scnt résumées dans l'organigramme suivant : 


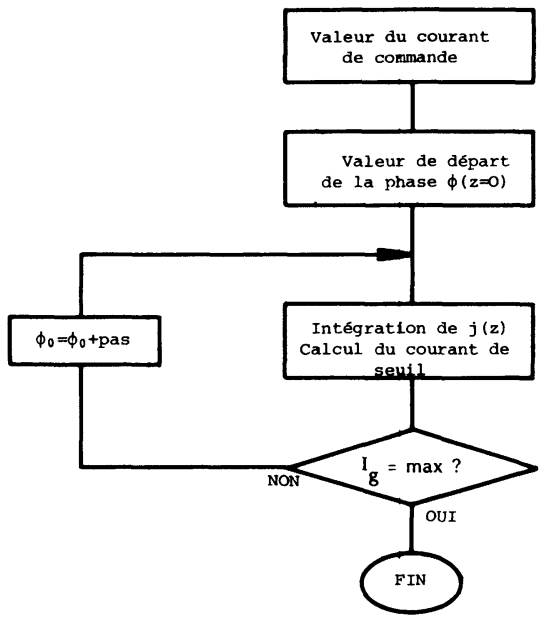

Fig. 5. - Calcul du courant de seuil d'une jonction élémentaire.

[Computation of the threshold current for an elementary junction.]

Lorsque le courant de seuil de chaque jonction élémentaire est déterminé, l'intégration sur la largeur de la jonction donne le courant de seuil total.

2.2 CAS DES JONCTIONS COURTES $\left(L<2 \lambda_{\mathrm{J}}\right)$. - Le champ magnétique créé par le courant dans la jonction (champ propre) est alors négligeable devant le champ de commande [2]; l'intégration de l'équation (7) donne dans ces conditions :

$$
\phi(y, z)=\frac{2 \pi d \mu_{0}}{\Phi_{0}} z H_{\mathrm{cy}}(y)+\phi_{(z=0)} .
$$

La relation (8) permet de calculer la densité locale de courant Josephson. Son intégration sur la surface de la jonction élémentaire et la maximisation du résultat par rapport à la phase $\phi_{(z=0)}$ donne le courant de seuil. Dans ce cas la phase $\phi_{(z=0)}$ est constante, elle vaut $\pi / 2$ et le courant de seuil pour une jonction élémentaire est donné [13] par la relation :

$$
\Delta I_{\mathrm{g}_{\max }}=j_{0} L \Delta y\left|\frac{\sin \frac{\pi \Phi(y)}{\Phi_{0}}}{\frac{\pi \Phi(y)}{\Phi_{0}}}\right|
$$

où $\Phi(y)=\mu_{0} d L H_{\mathrm{cy}}(y)$.

L'intégration sur la surface de la jonction conduit à :

$$
I_{\operatorname{gax}_{\max }}=j_{0} L \int_{0}^{W}\left|\frac{\sin \frac{\pi \Phi(y)}{\Phi_{0}}}{\frac{\pi \Phi(y)}{\Phi_{0}}}\right| \mathrm{d} y .
$$

Pratiquement on ne connaît pas la fonction $\Phi(y)$, mais seulement ses valeurs en un nombre fini de points. Une intégration numérique est encore nécessaire.

Le calcul d'une caractéristique de seuil pour une jonction de longueur quelconque est résumé sur la figure 6.

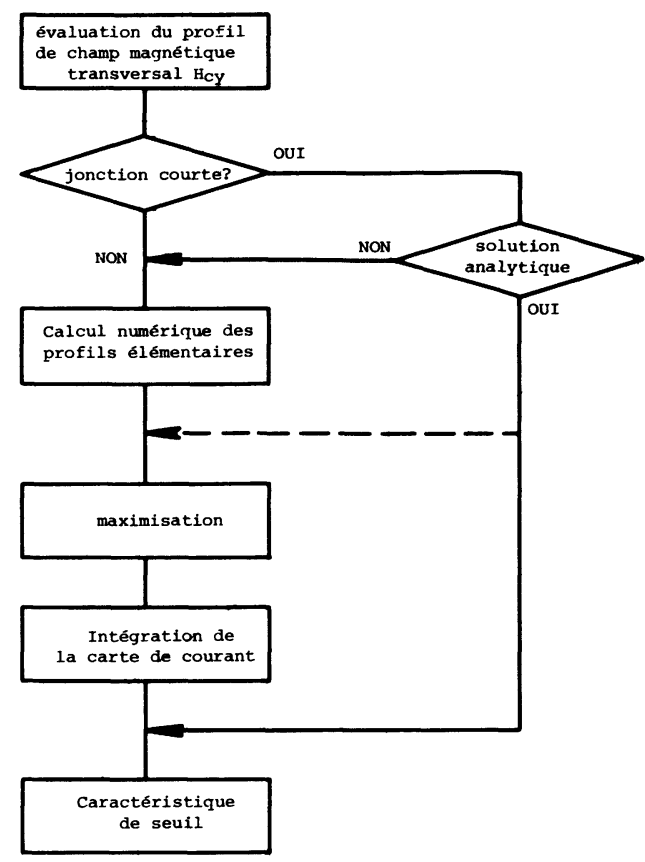

Fig. 6. - Calcul de la caractéristique de seuil dans le cas général.

[Computation of the threshold characteristic in the general case.]

La méthode présentée permet théoriquement de traiter des jonctions de longueur quelconque. Cependant, pour les grandes longueurs, la sensibilité du courant critique à la phase à l'origine est très grande et la précision requise sur le calcul numérique augmente beaucoup (pour $L / \lambda_{\mathrm{J}}=10$, il faut $\frac{\Delta \phi_{(z=0)}}{\phi_{(z=0)}} \simeq$ $\left.10^{-6}\right)$. De plus, la forme de la répartition de courant le long de la jonction est plus complexe pour les jonctions longues et le raccordement entre les jonctions élémentaires impose une partition de plus en plus fine. Pour cette raison, nous n'utilisons la méthode présentée ici que pour des jonctions courtes. Pour les jonctions longues nous développons actuellement une méthode numérique basée sur un principe de perturbation.

\section{Résultats de calcul.}

Le calcul des caractéristiques de seuil a été effectué pour les profils simplifiés de champ magnétique représentés en encart sur les figures 7 et 8.

Les courbes (2) des figures 7 et 8 représentent les caractéristiques de seuil calculées à partir des deux 

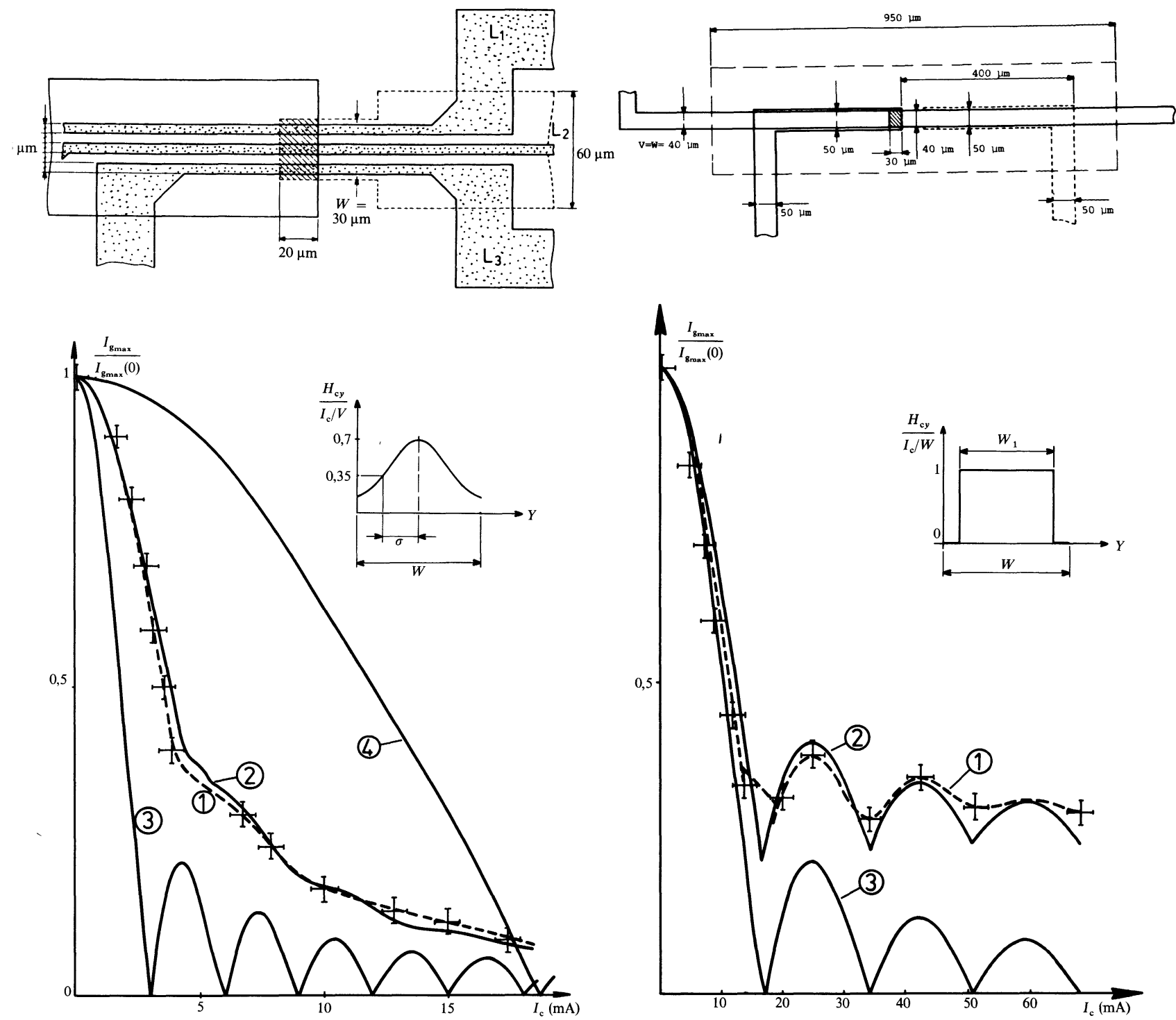

Fig. 7. - Caractéristiques de seuil de la jonction à commande multiple représentée ci-dessus. (1) Caractéristique expérimentale. (2) Simulation à l'aide du profil de champ en éncart. (3) Caractéristique idéale supposant un champ constant $\left(I_{\mathrm{c}} / V\right)$. (4) Caractéristique idéale supposant un champ constant $\left(I_{\mathrm{c}} / W\right) ; V$ : largeur des lignes de commande, $W$ : largeur de la contre-électrode, $\sigma:$ largeur à mi-hauteur de la courbe, $I_{\mathrm{c}}$ : courant de commande dans la ligne centrale.

[Threshold characteristics for the multiple command junction depicted here above. (1) Experiment. (2) Simulation by means of the field profile given in inset. (3) Ideal characteristic supposing a constant field $\left(I_{\mathrm{c}} / V\right)$. (4) Ideal characteristic supposing a constant field $\left(I_{\mathrm{c}} / W\right) ; V$ : width of the command lines, $W$ : width of the counter-electrode, $\sigma:$ half-height width of the magnetic field, $I_{\mathrm{c}}$ : command current in the central line.]

profils types. La comparaison de ces caractéristiques avec le cas où le champ magnétique est constant dans la jonction (cas idéal, courbe (3)), montre que l'in-

Fig. 8. - Caractéristiques de seuil de la jonction à commande unique représentée ci-dessus. (1) Caractéristique expérimentale. (2) Simulation (profil de champ en encart). (3) Caractéristique idéale supposant un champ constant idéal $\left(I_{\mathrm{c}} / W\right) ; W$ : largeur de la contre-électrode; $W_{1}$ : largeur de la zone de champ non nul.

[Threshold characteristics of the single command junction depicted here above. (1) Experiment. (2) Simulation (field profile in inset). (3) Ideal characteristic supposing an ideal constant field $\left(I_{\mathrm{c}} / W\right) ; W$ : width of the counter-electrode; $W_{1}$ : width of the non-zero field zone.]

homogénéité du champ magnétique a un double rôle :

- un relèvement du niveau des lobes secondaires lié à la faible valeur du champ magnétique dans certaines zones.(les bords de la jonction),

- un lissage des caractéristiques provoqué par les variations du champ magnétique autour de la valeur moyenne. 
Ces deux effets dégradent l'immunité au bruit et réduisent les marges de fonctionnement des dispositifs logiques.

Les courbes (1) des figures 7 et 8 représentent les caractéristiques expérimentales obtenues avec des jonctions de type $\mathrm{Nb}-\mathrm{Nb}_{2} \mathrm{O}_{5}-\mathrm{Pb}\left(\mathrm{I}_{n}\right)$ fabriquées par le LETI $\left({ }^{1}\right)$. Par ajustement des paramètres $\sigma$ et $W_{1}$ des profils de champ magnétique, nous obtenons un bon accord entre les résultats théoriques et expérimentaux. La comparaison entre ces résultats permet d'étalonner en amplitude les répartitions de champ magnétique utilisées. Pour le dispositif multicommandé de la figure 7 , la valeur moyenne du champ de commande $H_{\mathrm{c}_{\text {moy }}}=\frac{1}{W} \int_{0}^{W} H_{\mathrm{c} y} \mathrm{~d} y$ est comprise entre $I_{\mathrm{c}} / W$ et $I_{\mathrm{c}} / V\left(H_{\mathrm{c}_{\mathrm{moy}}}=3,2 \quad I_{\mathrm{c}} / W=0,5 \quad I_{\mathrm{c}} / V\right)$.

La valeur $I_{\mathrm{c}} / W$ correspond au cas idéal où les effets de bord et de proximité sont négligeables. La valeur $I_{\mathrm{c}} / V$ correspond au cas extrême où l'effet de blindage par la contre-électrode est inexistant [5].

Lorsque la ligne de commande est de même largeur que la contre-électrode (dispositif " in-line ») les effets de bord sont moins importants et les variations du champ de commande sont plus faibles. Pratiquement la largeur des lignes de commande est fixée par leur nombre et la largeur de la contre-électrode. On peut donc difficilement intervenir sur ce paramètre pour modifier l'effet de blindage. Par contre il est plus facile d'agir sur l'épaisseur de la contreélectrode. On ne peut toutefois pas trop l'augmenter car l'effet de blindage diminue également la valeur moyenne du champ de commande et par conséquent le gain du dispositif. Le choix de l'épaisseur de la contre-électrode doit donc faire l'objet d'un compromis entre l'efficacité des lignes de commande (gain

( $\left.{ }^{1}\right)$ Laboratoire d'Electronique et de Technologie de l'Informatique du Centre d'Etudes Nucléaires de Grenoble, France. de la cellule) et la réduction du niveau des lobes secondaires (immunité au bruit).

\section{Conclusion.}

La méthode développée nous a permis de tenir compte de l'inhomogénéité du champ magnétique de commande dans le calcul des caractéristiques de seuil des dispositifs Josephson. Elle est applicable dans le cas où les variations spatiales du champ de commande sont importantes et pour des jonctions courtes $\left(L \leqslant 2 \lambda_{\mathrm{J}}\right)$.

Par ajustement des paramètres des profils types utilisés, nous avons déduit la valeur moyenne réelle du champ magnétique de commande dans la jonction. Cette valeur est directement liée à l'effet de blindage de la contre-électrode et à la largeur de la ligne de commande. Elle est différente de la valeur généralement admise pour la simulation de tels dispositifs. L'analyse des résultats montre que l'inhomogénéité du champ de commande est à l'origine d'un relèvement du niveau des lobes secondaires et d'un lissage des caractéristiques de seuil. Il en résulte une réduction des marges de fonctionnement et de l'immunité au bruit qui peuvent provoquer des aléas de fonctionnement dans les circuits logiques.

L'inhomogénéité et la valeur moyenne du champ de commande sont liées à la largeur et à l'épaisseur de la contre-électrode et des lignes de commande. Pour limiter le relèvement des lobes secondaires, il faut homogénéiser le champ dans la jonction. On peut y parvenir en augmentant l'épaisseur de la contre-électrode ou en utilisant des matériaux à faible $\lambda_{\mathrm{L}}$. Cependant un effet de blindage important par la contre-électrode entraîne une diminution de la valeur moyenne du champ de commande et une réduction de l'efficacité des lignes de commande. Le choix de l'épaisseur de la contre-électrode doit donc se faire à partir d'un compromis sur les critères de gain et d'immunité au bruit.

\section{Bibliographie}

[1] Kautz, R. L., J. Appl. Phys. 49, no 1 (1978) 308-314.

[2] Matisoo, J., I.B.M. Journal 24, no2 (1980) 113-129.

[3] Gheewala, T. R., I.B.M. Journal 24, no 2 (1980) 130$142(1980)$.

[4] Owen, C. S., Scalapino, D. J., Phys. Rev. 164, no 2 (1967) 538-544.

[5] Matisoo, J., J. Appl. Phys. 40, no 4 (1969) 1813-1820.

[6] Basavaiah, S., Broom, R. F., IEEE Trans. Mag. Mag 11, No 2 (1975) 759-762.

[7] Barone, A., Johnson, W. J., Vaglio, R., J. Appl. Phys. 46, no 8 (1975) 3628-3632.
[8] Chilo, J., Monllor, C., Revue Phys. Appl. 17 (1982) 465-472.

[9] Angenieux, G., Thèse de 3e cycle, INP Grenoble (1982).

[10] Chilo, J., Monllor, C., IEEE Trans. Mag. Mag 19, $\mathrm{n}^{\circ} 3$ (1983) 1193-1195.

[11] Barone, A., Esposito, F., Likharev, K. K., Semenov, V. K., Todorov, B. N., Vaglio, R., J. Appl. Phys. 53, no 8 (1982) 5802-5809.

[12] Pace, S., Vaglio, R., J. Appl. Phys. 50, no 11 (1979) 7254-7255.

[13] Taylor, B. N., J. Appl. Phys. 39, no 6 (1968) 2490-2502. 